\title{
Prevention of adverse events in the administration of drugs
}

\author{
G Diano ${ }^{*}$, P Vrenna \\ From de Senectute: Age and Health Forum \\ Catanzaro, Italy. 5-7 December 2009
}

\section{Incorrect use of drugs may cause adverse events with serious consequences for patients.}

Adverse event ( $\underline{\text { Adverse events }})$ refers to an event related to the care process and which involves an injury to the patient, unintended and undesirable.

Errors in drug therapy (also called error of therapy) are predictable events that may cause or lead to the inappropriate use of the drug or a threat to hospitalized patients, which may occur during the process of managing the drug for prevention and therefore should be considered by the whole management system of healthcare.

These events, predictable and avoidable must be differentiated from the adverse drug reactions (ADRAdverse Drug Reaction), linked to the drug itself and that are detected and evaluated by pharmacovigilance.

The causes of errors in therapy are multifactorial and involve different health professionals.

Basically 5 categories of error are recognized .

- Error limitation

- Clerical error / interpretation,

- Error processing;

- Error distribution

- Administration error

\section{Error limitation}

The error of prescribing may relate to the decision to prescribe a drug, and the process of writing the prescription (quality and completeness of essential information).

\section{Clerical error / interpretation}

Errors occur when the prescription, while still written by hand, is not properly recorded on the therapy.

\section{Preparation errors}

After the prescription, preparation is the most critical phase in the process of administration of the drug in the hospital.

\section{Distribution}

The distribution of medication errors occur both when it is distributed by hospital pharmacy departments and clinics and as when it is distributed directly to patients.

\section{Administration}

The error of administration can be considered as a deviation between medication that your doctor has prescribed according to proper standards of clinical practice and the drug received by the patient.

\section{Conclusions}

Currently examining the data in the literature, it is clear that the strategies adopted to reduce errors in therapy are as follows:

1. Computerized systems for registration of therapy,

2. Distribution of drugs in unit doses (with different levels of self-medication),

3. Active participation of clinical pharmacist management of therapy.

Published: 19 May 2010

doi:10.1186/1471-2318-10-S1-L76

Cite this article as: Diano and Vrenna: Prevention of adverse events in the administration of drugs. BMC Geriatrics 2010 10(Suppl 1):L76.

Hospital ASP Civile Crotone 